\title{
A study of the avalanche-to- streamer transition in arbitrary gases by particle simulation
}

\section{Journal Article}

Author(s):

Rabie, Mohamed; Franck, Christian (D)

Publication date:

2016

Permanent link:

https://doi.org/10.3929/ethz-b-000115106

Rights / license:

In Copyright - Non-Commercial Use Permitted

Originally published in:

Journal of Physics D: Applied Physics 49(17), https://doi.org/10.1088/0022-3727/49/17/175202 
This is a post-refereeing copy of the manuscript submitted to IOP.

The original version can be found on the IOP website:

Digital Object Identifier: http://dx.doi.org/10.1088/0022-3727/49/17/175202 


\title{
A study of the avalanche-to-streamer transition in arbitrary gases by particle simulation
}

\author{
M. Rabie and C.M. Franck \\ Power Systems and High Voltage Laboratories, ETH Zurich, 8092 Zurich, Switzerland \\ E-mail: cfranck@ethz.ch
}

\begin{abstract}
.
We systematically investigate the avalanche-to-streamer transition (AST) over a wide range of pressures and homogenous background electric fields and for a comprehensive list of gases, namely pure nitrogen, carbon dioxide, oxygen, argon, sulfur hexafluoride and synthetic air. The discharge starts from an initial seed electron and is temporally followed from the avalanche regime, through the first significant distortion of the background field and the subsequent increasing deviation from the Gaussian electron density profile, up to the occurrence of runaway electrons accompanied by the sudden and dramatic increase of electron energy and electron number multiplication. We detect weak influence of the background electric field value and the gas composition, but strong influence of the gas density on the electron number at which the transition occurs. The simulations are performed by means of a fullyinteracting particle simulation program that combines a particle-in-cell/Monte Carlo collision model (PIC/MCC) with a three-dimensional Poisson solver in order to account for the space charge generated by the electrons and ions. The freely-available program is based on the METHES code and is universally applicable to arbitrary gas mixtures with complete cross section sets.
\end{abstract}

PACS numbers: 52.80.Dy 51.50.+v 52.25.Fi

Submitted to: J. Phys. D: Appl. Phys.

\section{Introduction}

In many cases, an electrical discharge starts with a single electron - or more precisely an ion/electron pair - that evolves into an avalanche and subsequently into a streamer [1]. The avalanche-to-streamer transition (AST) is crucial for the physics of lightning in the atmosphere [2] and for applications such as gaseous insulation for electrical equipment [3,4] or gaseous particle detectors [5]. It is particularly instructive to monitor the avalanche-to-streamer transition as the system's parameters, such as the electric field strength, ambient pressure and gas composition are changed. Cosmic rays may create free electrons that are the origin of lightning at various ambient pressures and temperatures in altitudes ranging from the troposphere to the mesosphere [6, 7]. In 
gaseous insulation systems, the pressure and the gas composition is typically optimized in order to suppress the streamer formation up to high electric fields [8, 9]. Gaseous particle detectors seek gas compositions and pressures that result in maximum signal gain and high time resolution while operating in the avalanche mode and preventing streamers [10, 11].

A precise model of the avalanche-to-streamer transition must include electron and ion kinetics dominated by collisions with the background gas and the interaction of the particle ensemble via its own space charge. Impact-ionization is the main electron source during the growth of an avalanche towards a streamer and electron production from photo-ionization is responsible for seed electrons outside the streamer head in certain gases [12]. Various definitions for the AST exist, which have in common that the transition is associated with the amplitude of the space charge electric field [3,13-16]. The widely-used criterions from Raether [13] and Meek [14, that is when the electric field generated by the space charge becomes comparable with the background electric field, greatly simplify the procedure for deriving the critical electron number at which the AST occurs. A correction to the Meek criterion in particular due to electron diffusion was conducted within a non-interacting fluid model [16]. The authors identify the transition point at the first significant distortion of the background field by the space charge, specifying "significant" as a maximal field enhancement of $3 \%$. However, fluid simulations cannot describe certain aspects of streamers such as runaway electrons [17. Therefore, particle models as well as hybrid models, which combine fluid and particle models, were used to investigate streamers, see e.g. [18]. First attempts to investigate discharges on the basis of particle-in-Cell/Monte Carlo collision (PIC/MCC) models were done for nitrogen within one-dimensional configuration space [19]. PIC/MCC models in two dimensions assuming cylindrical symmetry were used to investigate streamer formation in atmospheric pressure nitrogen [20 22], radio frequency discharges [23], and to analyze runaway electrons [24 26], space charge effects in resistive plate chambers [27] and point-to-plane corona discharge in nitrogen [28]. Furthermore, 3D $\mathrm{PIC}$ /MCC simulations were conducted in high-pressure electronegative gases with focus on laser-triggered gas switches [29]. The listed PIC/MCC simulations are based on different physical models and numerical methods, they use different definitions for the AST and their focus is on diverse physical phenomena in specific gases. To date, no comprehensive investigation by means of $\mathrm{PIC} / \mathrm{MCC}$ simulation is available that gives an overview of the AST for different gas species, ambient pressures and electric field strengths. In many applications, the AST occurs in strongly inhomogeneous background fields, e.g. close to sharp tips or rough surfaces. Due to the variety of possible inhomogeneous field configurations, the AST can be evaluated on a case-by-case basis only, whereas in homogenous background fields more universal statements regarding the transition are possible independent from the system's geometry.

In this work, we investigate the beginning of a discharge that emerges from a single low-energy electron in a homogenous background electric field. We follow the discharge that is growing due to impact-ionization towards a regime where the electrostatic 
interaction becomes relevant. We account for this interaction by means of mean field theory. The many-body problem of interacting electrons and ions is reduced to the Coulomb force on electrons arising from the space charge of the electron and ion densities. Magnetic coupling via the Lorentz Force is neglected. Clear transitions are identified on the basis of sudden changes in the discharge behavior and not by the widelyused method of monitoring the amplitude of the space charge electric field. Within a fully-interacting PIC/MCC simulation, the first transition, which distinguishes the noninteracting avalanche regime and the space charge affected regime, becomes manifest in an increase of electron diffusion. The second transition involves a sudden occurrence of a large fraction of high-energy (or runaway) electrons, and therefore a dramatic increase of the system's mean kinetic energy and electron number multiplication. Our final goal is to determine comprehensively the AST for different gas compositions, pressures and electric fields. The evolution from avalanche to streamer is systematically investigated for pure argon $(\mathrm{Ar})$, nitrogen $\left(\mathrm{N}_{2}\right)$, oxygen $\left(\mathrm{O}_{2}\right)$, carbon dioxide $\left(\mathrm{CO}_{2}\right)$, sulfur hexafluoride $\left(\mathrm{SF}_{6}\right)$ and synthetic air $\left(80 \% \mathrm{~N}_{2}, 20 \% \mathrm{O}_{2}\right.$ by volume). We place particular emphasis on providing a tool that is universally and easily applicable to arbitrary gas mixtures with complete cross section sets in the format provided by the LXCat database [30]. This makes it possible to directly compare the critical electron number at the AST for different gas compositions.

\section{Simulation methods}

The simulation program is based on the freely available Monte Carlo code METHES [31]. For arbitrary gas mixtures, electron transport parameters for steady-state conditions in the presence of uniform electric fields can be derived on the basis of input cross sections available on LXCat. Following the null-collision technique [32] and assuming isotropic scattering for electron-molecule collisions, it is possible to simultaneously trace up to $10^{6}-10^{7}$ electrons using a present-day standard computer. Simulations assume homogeneous gas particle number density $N$, which is deduced from the pressure $p$ and the temperature $T$ via the ideal gas law. The reduced electric field $E / N$, which is the ratio of the background electric field strength to $N$, is given in units of Townsend (Td), which is defined as $1 \mathrm{Td}=10^{-21} \mathrm{Vm}^{2}$.

The code has been described in detail and has been tested for various gases and gas mixtures in a previous work [31]. Several changes and extension of the original METHES code are necessary to account for space charge effects:

- extrapolating the cross sections to infinite energies $\varepsilon$, see section 2.1

- adding a functionality to derive particle densities from the limited amount of electrons provided by the computer, see section 2.2

- adding an efficient solver for the three-dimensional Poisson equation, see section 2.3

- integrating electron trajectories of relativistic energies in inhomogeneous fields (the 
time-integration of electron trajectories of the original METHES code was limited to low-energy electrons in homogenous electric fields), see section 2.4

\subsection{Cross section input}

The relevant types of electron-neutral collisions for the Monte Carlo simulation are elastic, inelastic, ionizing and attaching collisions. The collision data for argon Ar, nitrogen $\mathrm{N}_{2}$ and oxygen $\mathrm{O}_{2}$ [33], for carbon dioxide [34] and for sulfur hexafluoride $\mathrm{SF}_{6}$ [35] were downloaded from the LXCat database. In the present simulations, the pressure dependency of three-body attachment processes, such as the parent anion attachment in $\mathrm{O}_{2}$, is neglected. This means that the cross section is assumed to be independent from the pressure. Most cross sections that are available on the LXCat database are limited to energies $\varepsilon \lesssim 1 \mathrm{keV}$. Beyond this, we assume for all cross sections the limit of Coulomb scattering $\propto 1 / \varepsilon^{2}$, which corresponds to the first-order Born approximation for a screened Coulomb potential. This is generally valid due the rapid convergence of the Born series for high energies, see e.g. [36]. We ensure that the cross sections and their derivatives are continuous at the boundaries.

\subsection{Particle densities}

In order to account for space charge distorted fields, the Poisson equation must be solved and therefore the Monte Carlo Code is extended with a Particle-in-Cell (PIC) scheme to derive particle number densities. However, today's computers cannot efficiently handle the large electron numbers at the AST. To overcome this problem, the limited amount of electrons provided by the computer are used as "probes" to resolve spatio-temporally the ionization and attachment events accompanied by the changes in electron and ion densities. We introduce a maximum allowed number of electrons $N_{\max }$. The latter can be defined by the user and was set in the present work to $N_{\max }=10^{5}$ for reasons of computational efficiency. The electron number $\tilde{N}_{\max }$ just after $N_{\max }$ has been exceeded can be equal but generally is slightly larger than $N_{\max }$. From that moment on, the electron number is fixed to $\tilde{N}_{\text {max }}$ and these electrons are denoted as "probe electrons". Before the electron number reaches $N_{\max }$, real and probe electrons are equivalent and the densities of electrons $\rho_{\mathrm{e}}(\mathbf{r})$, cations $\rho_{+}(\mathbf{r})$ and anions $\rho_{-}(\mathbf{r})$ are straightforwardly obtained by mapping the particle positions onto the grid $\mathbf{r}=\left(x_{i}, y_{j}, z_{k}\right)$.

After exceeding $N_{\max }$, the number of probe electrons after an ionization event is kept fixed by removing a random electron from the ensemble. In case of an attachment event, the electron is removed and a random electron from the remaining ensemble is cloned in terms of position and energy. This technique has been described and extensively tested [31,37], ensuring the correct spatial and momentum distribution of the electron ensemble but not the correct magnitude of the particle number density. Therefore, a procedure for the charge densities is used in order to obtain the density of real electrons from the density of probe electrons: the current number of electrons $N_{\mathrm{e}}$, cations $N_{+}$and anions $N_{-}$are obtained by the spatial integral of the particle 
densities $N_{\mathrm{e},+,-}=\int \rho_{\mathrm{e},+,-}(\mathbf{r}) \mathrm{d}^{3} \mathbf{r}$. Subsequently, the numbers $N_{\mathrm{i}}$ and $N_{\mathrm{a}}$ of ionization and attachment events are counted and the corresponding increase in particle number during $\Delta t$ is calculated by $\Delta N_{+}=N_{\mathrm{e}} N_{\mathrm{i}} / \tilde{N}_{\max }$ for the cations, by $\Delta N_{-}=N_{\mathrm{e}} N_{\mathrm{a}} / \tilde{N}_{\max }$ for the anions and by $\Delta N_{\mathrm{e}}=\Delta N_{+}-\Delta N_{-}$for the electrons. Correspondingly, the particle densities change according to

$$
\rho_{\mathrm{e},+,-}(\mathbf{r}, t+\Delta t)=\rho_{\mathrm{e},+,-}(\mathbf{r}, t)+\frac{\Delta N_{\mathrm{e},+,-}}{N_{\mathrm{e}}} \rho_{\mathrm{e}}(\mathbf{r}, t)
$$

The number of ionization events must be much smaller than the fixed size of the electron ensemble used for the simulation. The constraint $N_{\mathrm{i}} / \tilde{N}_{\max }<10^{-2}$ is fulfilled by adjusting when necessary the time steps during the simulation by changing the maximum collision frequency. This is in particular important for obtaining the correct densities for avalanches in strong electric fields, where ionizing collisions dominate over elastic and excitation collisions. Ions are treated as immobile, since electron mobilities exceed ion mobilities by up to three orders of magnitude.

\subsection{Solving the Poisson equation on a moving grid}

The Poisson equation $\Delta \phi=\rho / \varepsilon_{0}$ for the electric potential $\phi$ is governed by the total charge density $\rho=\rho_{\mathrm{e}}+\rho_{+}+\rho_{-}$. Considering a particle ensemble far away from the boundaries, the total electric field $\mathbf{E}$ is the superposition of the uniform background field $\mathbf{E}_{\mathrm{B}}$ and the field originating from the space charge $\mathbf{E}=\mathbf{E}_{\mathrm{B}}-\nabla \phi$. Boundary conditions are therefore not imposed onto the solution, which is derived by means of a Jacobi relaxation method on a three-dimensional cartesian grid $\left(x_{i}, y_{j}, z_{k}\right)$ with spacing $\Delta x, \Delta y, \Delta z$. The potential $\phi_{i, j, k}$ is expressed on the grid point $\left(x_{i}, y_{j}, z_{k}\right)$ by the local charge density $\rho_{i, j, k}$ and the potentials on the neighbouring sites via

$$
\begin{aligned}
\phi_{i, j, k}= & \frac{1}{2} \frac{\Delta x^{2} \Delta y^{2} \Delta z^{2}}{\Delta y^{2} \Delta z^{2}+\Delta x^{2} \Delta z^{2}+\Delta x^{2} \Delta y^{2}}\left(\frac{1}{\Delta x^{2}}\left(\phi_{i+1, j, k}-\phi_{i-1, j, k}\right)\right. \\
& \left.+\frac{1}{\Delta y^{2}}\left(\phi_{i, j+1, k}-\phi_{i, j-1, k}\right)+\frac{1}{\Delta z^{2}}\left(\phi_{i, j, k+1}-\phi_{i, j, k-1}\right)+\rho_{i, j, k} / \epsilon_{0}\right) .
\end{aligned}
$$

This explicit seven-point difference scheme gives an approximate solution $\phi_{i, j, k}^{(p)}$ by applying equation (2) iteratively $p$-times on an initial potential $\phi_{i, j, k}^{(0)}$. The change in electron and ion densities during a time step $\Delta t$ is below $1 \%$ (see section 2.21) and hence the change in $\phi$. Therefore, by taking the previous potential $\phi(t-\Delta t)$ as initial guess, only a few iterations are needed to derive the potential $\phi(t)$ within $1 \%$ absolute accuracy. Thus, multigrid methods [38] which improve convergence of the solution are not needed for our simulations, neither are adaptive grid refinement techniques [39] which were introduced to follow the streamer evolution after branching on the basis of fluid models. Compared to direct solvers, the iterative Poisson solver has, besides speed, the advantage of suppressing electrostatic fluctuations from individual particles.

During the simulation, the grid encloses the entire region of the static ions and the moving electrons to ensure a fine spatial resolution in the relevant region. After each 
time step the grid $\left(x_{i}, y_{j}, z_{k}\right)$ is redefined and the potential as well as particle densities are linearly interpolated onto the new grid $\left(x_{i}^{\prime}, y_{j}^{\prime}, z_{k}^{\prime}\right)$, keeping particle numbers constant by normalizing the densities via

$$
\rho_{\mathrm{e},+,-}\left(\mathbf{r}^{\prime}\right)=\frac{\int \rho_{\mathrm{e},+,-}(\mathbf{r}) \mathrm{d}^{3} \mathbf{r}}{\int \rho_{\mathrm{e},+,-}\left(\mathbf{r}^{\prime}\right) \mathrm{d}^{3} \mathbf{r}^{\prime}} \rho_{\mathrm{e},+,-}(\mathbf{r}) .
$$

\subsection{Electron trajectories}

Due to the space charge, electrons are moving in a temporally and spatially varying electric field $\mathbf{E}$. As mentioned in section 2.3, the relative change of $\phi$ during $\Delta t$ is small. However, strong spatial variation of $\mathbf{E}$ as well as electron energies in the order of $\mathrm{keV}$ are characteristic for the AST. Such energies correspond to velocities $\mathbf{v}$ of $|\mathbf{v}| / c \approx 0.1$, where $c$ is the speed of light [40]. Accounting for the spatially varying electric field and the relativistic mass increase of the electrons we solve the equation of motion for the electron position $\mathbf{x}$ and the relativistic momentum $\mathbf{p}=m_{\mathrm{e}} \mathbf{v} / \sqrt{1-|\mathbf{v}|^{2} / c^{2}}$, where $m_{\mathrm{e}}$ is the electron mass. For each electron, the coupled system of differential equations

$$
\begin{aligned}
& \frac{d \mathbf{p}}{d t}=e \mathbf{E}, \\
& \frac{d \mathbf{r}}{d t}=\frac{\mathbf{p}}{\sqrt{m_{\mathrm{e}}^{2}+\mathbf{p}^{2} / c^{2}}}
\end{aligned}
$$

is integrated in the interval $[t, t+\Delta t]$ using $M A T L A B$ 's build-in solver ode45, which is based on an explicit Runge-Kutta $(4,5)$ formula [41. Here $e$ is the electron charge. For moderate electric fields and small space charge, inhomogeneities in $\mathbf{E}$ and relativistic effects are negligible and, instead of equations (4) and (5), Newtons second law of motion is applied to avoid time-consuming integration.

\section{Characterization of the AST}

The maximal field enhancement $\gamma=\left(E_{\max }-\left|\mathbf{E}_{\mathrm{B}}\right|\right) /\left|\mathbf{E}_{\mathrm{B}}\right|$ compares $E_{\max }$, which is the peak value of $|\mathbf{E}|$, with the background field $\mathbf{E}_{\mathrm{B}}$. It can be used as a measure for the significance of the space charge. However, values of $\gamma$ at the AST vary between $3 \%$ [16] and $100 \%$ [22]. In order to characterize the transition more precisely, we monitor averaged quantities of the electron's phase-space density and investigate the discharge based on the behavior of these quantities. The latter are the electron number $N_{\mathrm{e}}$, mean kinetic energy $\langle\varepsilon\rangle$, mean electron position $\langle\mathbf{r}\rangle$ and the standard deviation of $\mathbf{r}, \sigma=\left\langle(\mathbf{r}-\langle\mathbf{r}\rangle)^{2}\right\rangle^{1 / 2}$. For a non-interacting avalanche $\sigma$ corresponds to the width of the gaussian electron density profile in $\mathrm{x}-, \mathrm{y}$ - and $\mathrm{z}$-direction. Figure 1 shows the temporal evolution of the quantities that are used for the identification of the different regimes of a discharge. The avalanche (or swarm) regime is characterized by a constant $\langle\varepsilon\rangle$ as well as a linear increase of $\log \left(N_{\mathrm{e}}\right)$ and $\sigma_{\|}^{2}$ versus time, where $\sigma_{\|}$is the longitudinal component of $\sigma$ into the direction of $\mathbf{E}_{\mathrm{B}}$. This situation corresponds to temporally constant fluid parameters, namely effective ionization rate $\nu_{\text {eff }}$ and longitudinal diffusion constant $D_{\|}$. 


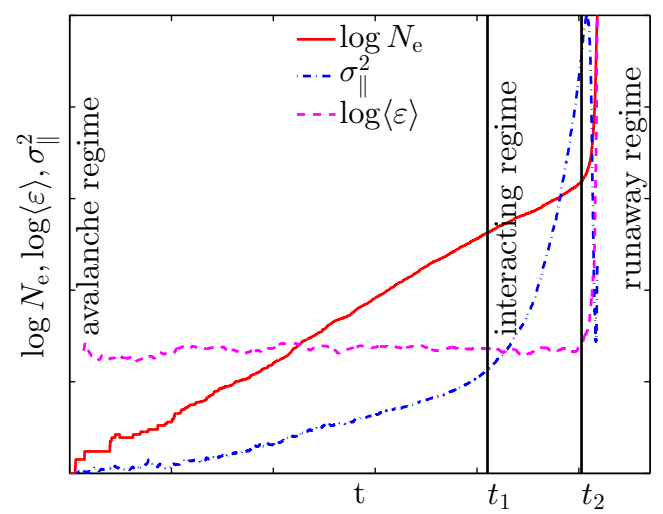

Figure 1: A typical temporal evolution of a discharge starting from a single electron with initially zero kinetic energy, in terms of electron number $N_{\mathrm{e}}$, mean kinetic energy $\langle\varepsilon\rangle$ and longitudinal width $\sigma_{\|}$(in arbitrary units). During transition 1 at time $t_{1}$, the avalanche starts to interact via its own space charge and $\sigma_{\|}^{2}$ becomes non-linear. Transition 2 at time $t_{2}$ is accompanied by the abrupt increase of $\langle\varepsilon\rangle$ and $N_{\mathrm{e}}$ by several orders of magnitude.

The most sensitive parameter with respect to the space charge is $\sigma_{\|}$. The clear but rather smooth transition of $\sigma_{\|}^{2}$ from linearity to non-linearity defines transition 1 - at time $t_{1}$ and electron number $N_{1}=N_{\mathrm{e}}\left(t_{1}\right)$ - and separates the interacting regime from the previous avalanche regime. The transversal component $\sigma_{\perp}^{2}$ and the mean position $\langle\mathbf{r}\rangle$ of the discharge, which are not shown in the figure, leave the linear regime with slight delay and less pronounced. In terms of fluid parameters, the interacting regime is accompanied by a strong increase of the longitudinal and transversal diffusion constants $D_{\|}$and $D_{\perp}$ and a slight decrease of $\langle\varepsilon\rangle$ and $\nu_{\text {eff }}$ compared to the avalanche regime. A second and more pronounced transition 2 takes place - at time $t_{2}$ and $N_{2}=N_{\mathrm{e}}\left(t_{2}\right)$ - in the form of a sudden and dramatic increase of $\langle\varepsilon\rangle$ and $N_{\mathrm{e}}$. This point in time clearly marks a transition from a regime that is influenced by space charge to a runaway regime. The method that we used to identify the transition points from the $\sigma_{\|}^{2}$-vs-time and the $\log \left(N_{\mathrm{e}}\right)$-vs-time curves is given in the appendix 8.1 ,

Figure 2(a) shows the maximal field enhancement $\gamma$ at selected instances for atmospheric pressure air. A value of $\gamma=3 \%$ is reached at an electron number of $N_{\mathrm{e}}=1.6 \cdot 10^{5}$ around $0.1 \mathrm{~ns}$ before transition 1 , which occurs at $N_{\mathrm{e}}=3.4 \cdot 10^{6}$. The values $\gamma=100 \%$ and $\gamma=200 \%$ are reached between $t_{1}$ and $t_{2}$ at $N_{\mathrm{e}}=2.1 \cdot 10^{7}$ and $N_{\mathrm{e}}=3.8 \cdot 10^{7}$. Transition 2 corresponds to $N_{\mathrm{e}}=4.8 \cdot 10^{7}$. The value of $\gamma=1000 \%$ occurs at $t=0.65$ ns and $N_{\mathrm{e}}=1.8 \cdot 10^{8}$. The corresponding radially integrated electron densities in longitudinal direction are shown in figure 2(b). For $\gamma=3 \%, \rho_{\mathrm{e}}$ is well described by a Gaussian, whereas subsequently for $\gamma=100 \%$ and $\gamma=200 \%$ the deviation from the Gaussian density profile can clearly be observed. 

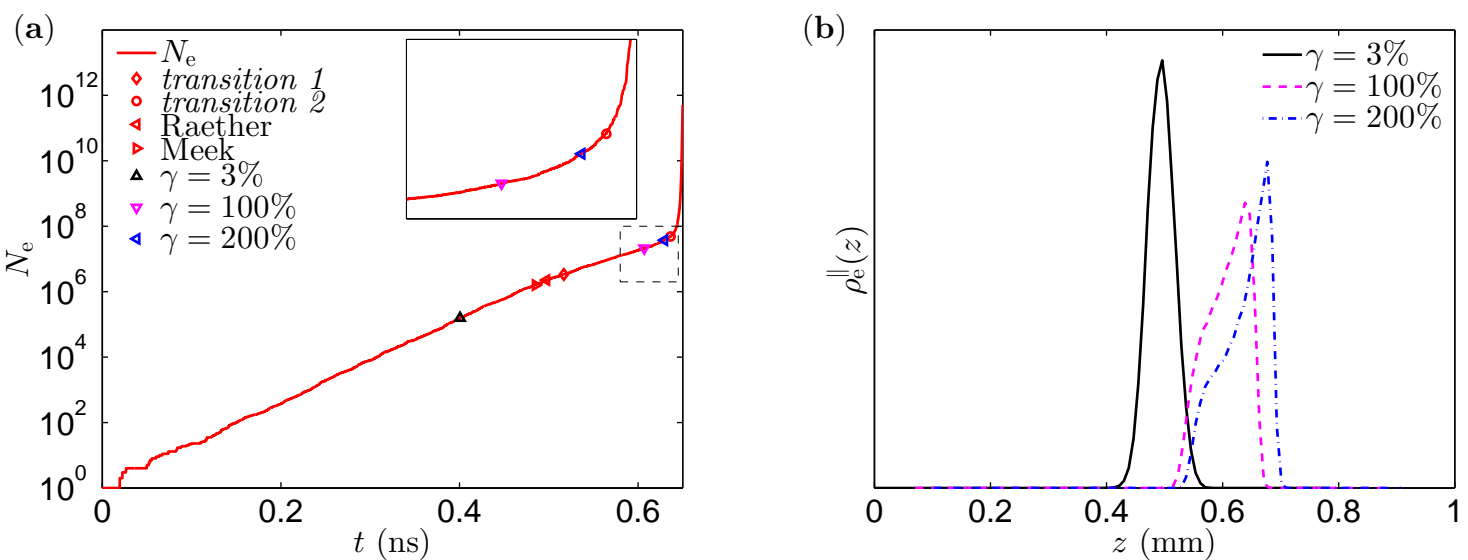

Figure 2: (a) Electron number vs time of a discharge, starting from a single electron with initially zero kinetic energy, in synthetic air at $E / N=400 \mathrm{Td}, p=100 \mathrm{kPa}$ and room temperature. The points corresponding to transition 1, transition 2, Raether criterion (eq. (6) ), Meek criterion (eq. (7)) and field enhancements of $\gamma=3,100$ and $200 \%$ are indicated with markers. (b) Spatial electron density profiles in direction of the background field $\mathbf{E}_{\mathrm{B}}$ at the instances where $\gamma=3,100$ and $200 \%$. Here, $\rho_{\mathrm{e}}^{\|}(z)=$ $\int \rho_{\mathrm{e}}(\mathbf{r}) d r_{\perp}$ is obtained by integrating the density $\rho_{\mathrm{e}}$ over the cartesian coordinates transversal to $\mathbf{E}_{\mathrm{B}}$. For easier comparison $\int \rho_{\mathrm{e}}^{\|}(z) d z$ is normalized to 1 .

\section{Results}

Simulations in $\mathrm{Ar}, \mathrm{N}_{2}, \mathrm{O}_{2}, \mathrm{CO}_{2}, \mathrm{SF}_{6}$ and synthetic air are performed at room temperature $(T=300 \mathrm{~K})$ with the cross section sets given in section 2.1 and the methods described in section 2.2 - 2.4. Numerical tests for different grid spacing and maximum allowed number of electrons $N_{\max }$ have been performed, see appendix 8.2 . For the present investigations, a number of grid nodes of $[x, y, z]=100 \times 100 \times 100$ and $N_{\max }=10^{5}$ was used. The energy and position of the initial electron was set to zero. For the pressures of $10 \mathrm{kPa}, 100 \mathrm{kPa}$ and $1000 \mathrm{kPa}$, we obtain the temporal evolution of electron avalanches up to the runaway regime for reduced fields in the range $200 \leq E / N \leq 2000$. The points $\left(t_{1}, N_{1}\right)$ of transition 1 and $\left(t_{2}, N_{2}\right)$ of transition 2 are identified from the temporal behavior of $\sigma_{\|}^{2}$ and $\log \left(N_{\mathrm{e}}\right)$, as described in section 3. Figure 3(a) shows the temporal evolution of $N_{\mathrm{e}}$ and the points of transition 1 and transition 2 for the investigated gas species at $700 \mathrm{Td}$ and $1000 \mathrm{kPa}$. The slope of $N_{\mathrm{e}}$ in the avalanche regime corresponds to the effective ionization rate $\nu_{\text {eff }}$ in the specific gas and for the present $E / N$-value. It increases in the order: $\mathrm{SF}_{6}, \mathrm{~N}_{2}$, synthetic air, $\mathrm{CO}_{2}, \mathrm{Ar}$ and $\mathrm{O}_{2}$. The electron numbers $N_{1}$ and $N_{2}$ do not show significant dependency on the gas species. Correspondingly, the times $t_{1}$ and $t_{2}$ of transition 1 and transition 2 approximately behave as $\propto \nu_{\text {eff }}^{-1}$. Figure 3(b) shows the temporal evolution of $N_{\mathrm{e}}$ in synthetic air for selected $E / N$-values. For increasing $E / N$-values $\nu_{\text {eff }}$ increases in the avalanche regime. No significant influence of the $E / N$-value on $N_{1}$ and $N_{2}$ is detected. 
Figure 3(c) shows the temporal evolution of $N_{\mathrm{e}}$ and transition 1 and transition 2 in synthetic air at $E / N=700 \mathrm{Td}$ and different pressures. The values $N N_{1}, N N_{2}, N t_{1}$ and $N t_{2}$ are basically pressure-independent, resulting in a $N^{-1}$ tendency of $N_{1}, N_{2}, t_{1}$ and $t_{2}$. The shift of the AST at $10 \mathrm{kPa}$ to higher $N t$ is solely due to the large variance of the MCC method for small numbers of electrons: by accident the first ionization collision of the initial electron happens relatively late and thus the buildup of the avalanche is delayed. With increasing electron number the variance of the MCC method decreases and the discharge propagates similar for all pressures independently from statistics.

Figure 4 summarizes the critical electron numbers of transition 1 and transition 2 for the investigated gases and pressures as a function of $E / N$. Similar to the selected cases shown in Figure 3, $N_{1}$ and $N_{2}$ decrease as $p$ increases and are almost constant with respect to $E / N$ for all investigated gases. For $E / N \lesssim 500, N_{1}$ increases significantly with decreasing $E / N$-values. In general, the dependency of $N_{1}$ and $N_{2}$ on the gas species is small and only slight variations of $N_{1}$ and $N_{2}$ is observed for different gas species. For comparison, the Raether- as well as Meek-criterion is evaluated for the same conditions, which will be described in more detail in section 5, Furthermore, for atmospheric pressure air data from a fluid model is presented [16]. 

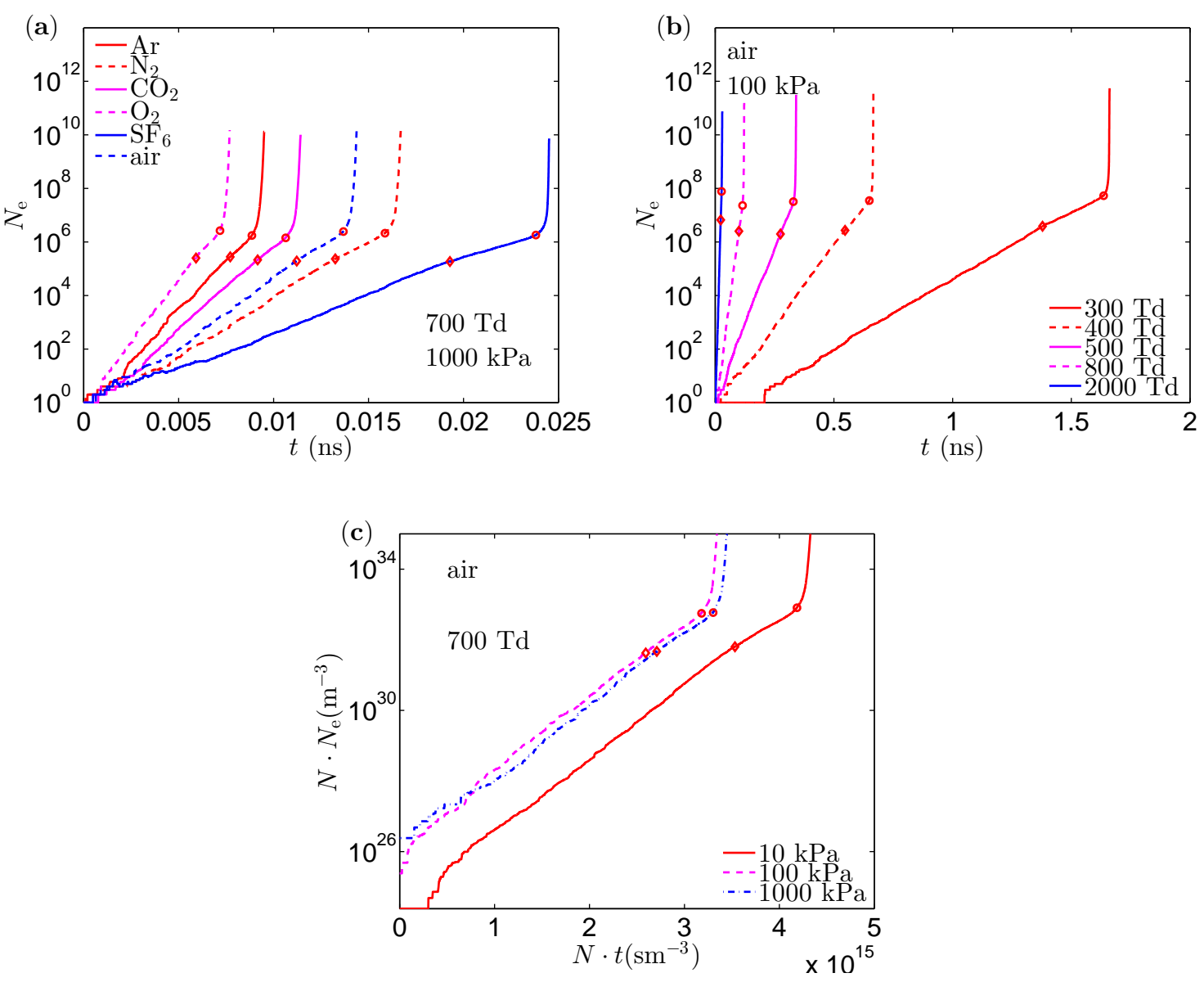

Figure 3: Electron number vs time for single-electron initiated discharges at room temperature. Electron numbers $N_{1}$ at time $t_{1}(\diamond)$ and $N_{2}$ at time $t_{2}(\circ)$. Results for (a) different gases at $p=1000 \mathrm{kPa}$ and $E / N=700 \mathrm{Td}$, (b) synthetic air at $p=100 \mathrm{kPa}$ and different $E / N$-values, and (c) synthetic air at $E / N=700 \mathrm{Td}$ and different pressures. In (c) the $\mathrm{x}$ - and $\mathrm{y}$ - axis are multiplied by the gas particle number density $N$.

\section{Discussion}

Our simulations of single-electron initiated discharges in different gases show that the temporal parameters $\langle\varepsilon\rangle, N_{\mathrm{e}}$ and $\sigma_{\|}$as well as the spatial electron density $\rho_{\mathrm{e}}$ are affected by the electric field generated by electrons and ions. Transition 1 separates the avalanche from the interacting regime as illustrated in figure 1. It was identified in section 3 by the point in time when $\sigma_{\|}^{2}$ starts to deviate from the linear behavior during the avalanche regime. This corresponds to an increase of the longitudinal diffusion constant $D_{\|}=1 / 2 \cdot d\left(\sigma_{\|}^{2}\right) / d t$ and has been observed previously in nitrogen by means of MCCsimulations [21]. The slight decrease of $\langle\varepsilon\rangle$ and the slope of $\log \left(N_{\mathrm{e}}\right)$, and thus $\nu_{\text {eff }}$, is in accordance with the experimental studies of Raether, who observed that the avalanche growth was weakened after the avalanche reaching around $10^{6}$ electrons, followed by a rise in current and breakdown at around $10^{8}$ electrons. In our investigations $\langle\varepsilon\rangle$ and 
$\nu_{\text {eff }}$ are reduced by a few percents only, whereas a drop of more than $50 \%$ of the same quantities is reported in nitrogen at $300 \mathrm{Td}$ [21]. The use of different cross section sets between the present investigations and Ref. [21] cannot explain this large discrepancy. The reason for the discrepancy might be due to the simulations methods used by the authors. For solving the Poisson equation, cylindrical symmetry and a 2D-grid of $6 \times 6$ grid points is applied. This grid might be too coarse for the precise spatial mapping of the sharp electron swarm and the long ion tail. Furthermore electron trajectories are calculated classically and the treatment of electron trajectories in inhomogeneous fields is not described by the authors.

As can be seen from figure 2, the interacting regime is accompanied by the evolution of $\rho_{\mathrm{e}}$ from Gaussian to non-Gaussian profile. Such non-Gaussian density profiles have been observed in MCC-simulations in atmospheric pressure nitrogen [21,22]. This spatial redistribution of the electron density is a consequence of reduced $\mathbf{E}$, and thus reduced ionization, in the center of mass of the electron ensemble and enhanced $\mathbf{E}$, and thus enhanced ionization, in front of the electron ensemble. This might be the reason why $\sigma_{\|}$is more affected by the space charge than $\sigma_{\perp}$, as described in section 3 ,

The dramatic increase of $\langle\varepsilon\rangle$ at transition 2 is due to the sudden appearance of a large fraction of runaway electrons. They are characterized by kinetic energies in a range where the frictional force due to collisions is too small to compensate the energy gain in the electric field. Depending on the amplitude of the background field $\mathbf{E}_{\mathrm{B}}$, electrons of energies $\varepsilon \gtrsim 0.1-1 \mathrm{keV}$ do not reach equilibrium anymore, and thus "run away" [24, 40, 42]. As described in section 4 and can be seen from Figure 2(a), in the case of synthetic air at $400 \mathrm{Td}$ the local field is enhanced up to a factor of $\sim 2-10$ in the immediate vicinity of transition 2. This corresponds to an enhanced local field of $\sim 8-40 E_{\mathrm{c}}$ where $E_{\mathrm{c}} / N \approx 100 \mathrm{Td}$ is the critical field of air. The occurrence of runway electrons at these fields is in agreement with the MCC-studies from Ref. [24], which indicated that "the $\sim 10 E_{\mathrm{c}}$ fields are able to accelerate a fraction of low-energy streamer tip electrons (several $\mathrm{eV}$ ) to energies of $\sim 2-8 \mathrm{keV}$ ". Generally, transition 2, and accordingly a fraction of runaway electrons, is observed over the investigated pressure range and for all $E>E_{\mathrm{c}}$. The finding that "there is a finite probability that a cold electron will be accelerated into the runaway regime, even at field values approaching $E_{\mathrm{c}}$ " has been shown in air by means of MCC/PIC simulations previously [26].

The comprehensive analysis of transition 1 and transition 2 makes it possible to compare the critical electron numbers $N_{1}$ and $N_{2}$ between different gas species, pressures and reduced electric fields $E / N$, see figure 3 and 4 . The influence of the gas species and the $E / N$-value on $N_{1}$ and $N_{2}$ is relatively small. The critical electron numbers $N_{1}$ and $N_{2}$ and times $t_{1}$ and $t_{2}$ scale with $\propto N^{-1}$ according to the similarity laws for streamer breakdown [43]. We compared our results with the expression for the streamer criterion from Raether 3 .

$$
\alpha x_{\mathrm{c}}=18.4+\ln \left(x_{\mathrm{c}}\right),
$$



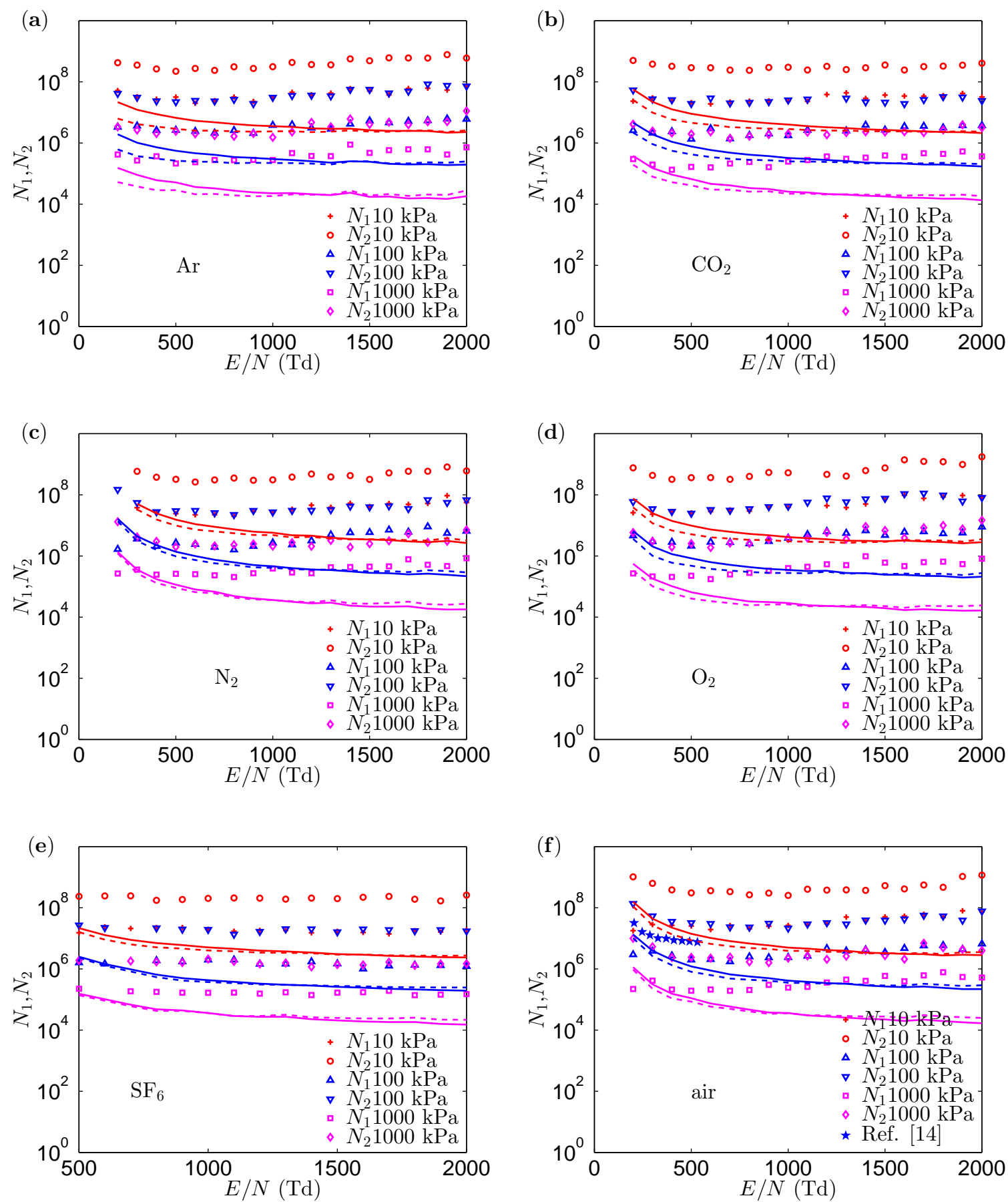

Figure 4: Electron numbers $N_{1}$ at transition 1 and $N_{2}$ at transition 2 vs $E / N$ for pressures of $10 \mathrm{kPa}, 100 \mathrm{kPa}$ and $1000 \mathrm{kPa}$ at room temperature for (a) argon, (b) carbon dioxide, (c) nitrogen, (d) oxygen, (e) sulfur hexafluoride and (f) synthetic air. For comparison, the values derived from the Raether criterion given by equation (6) $(-)$, Meek criterion given by equation (7) (- - -) and Ref. [16] for atmospheric pressure air (corresponding to the squares in Figure 6 of their manuscript). 
and from Meek [14

$$
\alpha x_{\mathrm{c}}+\ln (\alpha / p)=14.46+\ln (E / p)-1 / 2 \ln \left(p x_{\mathrm{c}}\right)+\ln \left(x_{\mathrm{c}}\right) .
$$

Here, $\alpha$ is the effective ionization coefficient and $x_{\mathrm{c}}$ is the length of the avalanche path along the background field $\mathbf{E}$ when it reaches the critical size. The coefficient $\alpha=\nu_{\text {eff }} / w$ ( $w$ is the electron drift velocity) were obtained from our simulations during the avalanche regime, and equations (6) and (7) were numerically solved for the individual gas species, fields and pressures. As can bee seen in figure 4, Raether's and Meek's criteria are both not reproducing our values for $N_{1}$ and $N_{2}$ and the disagreement is becoming more distinct as $E / N$ increases.

Other data exist for the case of atmospheric pressure $\mathrm{N}_{2}$ at around $200 \mathrm{Td}$, where the streamer was reached with $N_{\mathrm{e}} \approx 3.9 \cdot 10^{6}$ within MCC simulations [22]. This value is in between the identified electron numbers of $N_{1}=1.7 \cdot 10^{6}$ and $N_{2}=1.5 \cdot 10^{8}$ at $200 \mathrm{Td}$. In contrast, the gas pressure has a very pronounced effect on $N_{1}$ and $N_{2}$. The dependency of the AST on the pressure is qualitatively in agreement with the $N^{-1}$ scaling for streamer breakdown [43], the Raether- and Meek- criteria as well as with Ref. [16], where the authors found that in the case of high background field and small diffusion the critical electron number can be reduced by several orders of magnitude. This is consistent with the physical picture, that the effect of space charge sets in earlier at high pressures (for constant $E / N$ ) due to the combination of the increased ionization rates and the suppressed diffusion, which leads to high electron and ion densities and thus high electric fields. The same authors investigated the streamer criterion in synthetic air over a wide $E / N$-range. For $E / N$-values close to $E_{\mathrm{c}} \approx 100 \mathrm{Td}$, the critical electron number increases up to $N_{\mathrm{e}} \approx 1 \cdot 10^{9}$. This is not in the parameter range of our simulations: the lower boundary of the $E / N$-range in our work arises from the huge computation times $\propto \nu_{\text {eff }}^{-1}$ close to the critical field when $\nu_{\text {eff }} \rightarrow 0$.

Compared to our results, very small values for the critical electron number at the streamer criterion were obtained from breakdown experiments in homogenous fields in $\mathrm{SF}_{6}\left(N_{\mathrm{e}}=3.6 \cdot 10^{4}\right)$ [15] and synthetic air $N_{\mathrm{e}}=9.4 \cdot 10^{3}$ [44. The authors fitted breakdown voltages with a Paschen curve that is based on a simplified version of the streamer criterion from Raether, where $N_{\mathrm{e}}$ is assumed independent from the pressure. From the knowledge of the critical field (where $\nu_{\text {eff }}=0$ ) and its derivative $d \nu_{\text {eff }} / d E$ at this point, $N_{\mathrm{e}}$ could be extracted. Due to the exponential character of the avalanche growth, this evaluation of $N_{\mathrm{e}}$ is very sensitive to the measured Paschen curve and swarm parameter as well as to the field inhomogeneity. For example, an error of $20 \%$ in $\nu_{\text {eff }}$ due to an inaccurately measured breakdown voltage or due to a distorted electric field would modify the evaluated value of $N_{\mathrm{e}}$ by a factor of $\sim 10-20$. In addition, electron release from the electrode surfaces via secondary processes can reduce the breakdown voltage and consequently the assumed $\nu_{\text {eff }}$ and thus the obtained value of $N_{\mathrm{e}}$ from such experiments.

Finally, we note that in this contribution we deduced the gas particle number density $N$ from the pressure $p$ and the temperature $T$ via the ideal gas law. This is 
not correct for non-ideal gases, which e.g. are of relevance at filling pressures of high voltage apparatus [45]. However, we chose the variables $p$ and $T$, since they are typically measured in experiments, whereas $N$ is necessary for the MCC simulation.

\section{Summary and Outlook}

By means of particle simulation we comprehensively investigated the avalanche-tostreamer transition in homogeneous electric fields in pure nitrogen, carbon dioxide, oxygen, argon, sulfur hexafluoride and synthetic air for different gas densities. Discharge parameters, namely the temporal electron number and the width of the electron ensemble along the background electric field, were used to obtain well-defined transition points. We detected relatively small influence of the transition points on the reduced electric field strength and the gas species, but strong influence on the gas density. Critical electron numbers differ from those obtained by the Raether- and Meek- criterion by up to two orders of magnitude. We recognize the future need for investigations to resolve the large discrepancy between the results from the particle collision model, the widely-used streamer criteria and the few available experiments. Our simulation program can be downloaded from www.lxcat.net/download/METHES/ and can be used to investigate the avalanche-to-streamer transition in arbitrary gas mixtures. For practical applications, it could be beneficial to evaluate the avalanche-to-streamer transition in inhomogeneous background fields occurring e.g. close to sharp tips or rough surfaces. The possibility of using inhomogeneous background fields for arbitrary configurations could extend the relevance of the present simulation code to further practical use.

\section{Acknowledgement}

This work is financially supported by ALSTOM Grid GmbH, Pfiffner AG and ABB Switzerland.

\section{Appendix}

\subsection{Turning-point in a curve}

Figure 5 illustrates the method that is used for determining $t_{2}$ from the $N_{\mathrm{e}}$-curve. Similarly, $t_{1}$ is determined from the $\sigma^{2}$-curve, which is not shown. The transition point $\mathbf{p}$ maximizes $\mathbf{d}=|\mathbf{p}-(\mathbf{p} \cdot \hat{\mathbf{e}}) \hat{\mathbf{e}}|$, with $\hat{\mathbf{e}}$ being the unit vector along the connection line. In other words, $\mathbf{p}$ is geometrically identified as the point with the largest distance from the line that connects the first and end point of the curve. The point $\mathbf{p}$ is not sensitive to the chosen starting and end point of the curve. 


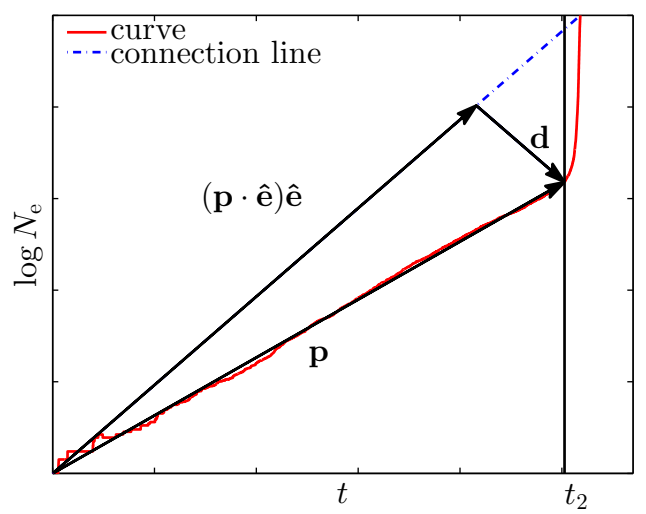

Figure 5: Method of determining the turning-point in a curve, here in particular the point at time $t_{2}$ from $\log \left(N_{\mathrm{e}}\right)$ vs time. Comment: $\hat{\mathbf{e}}$ and $\mathbf{d}$ appear not normal due to the different scaling of the $\mathrm{x}$ - and $\mathrm{y}$-axes.

\subsection{Numerical settings}

The sensitivity of the simulation results on different numerical settings are tested. Figure [6] shows the results for critical electron numbers for different numbers of probe electrons $N_{\text {max }}$ and different number of grid nodes. No significant effect of these parameters on simulation results is observed.

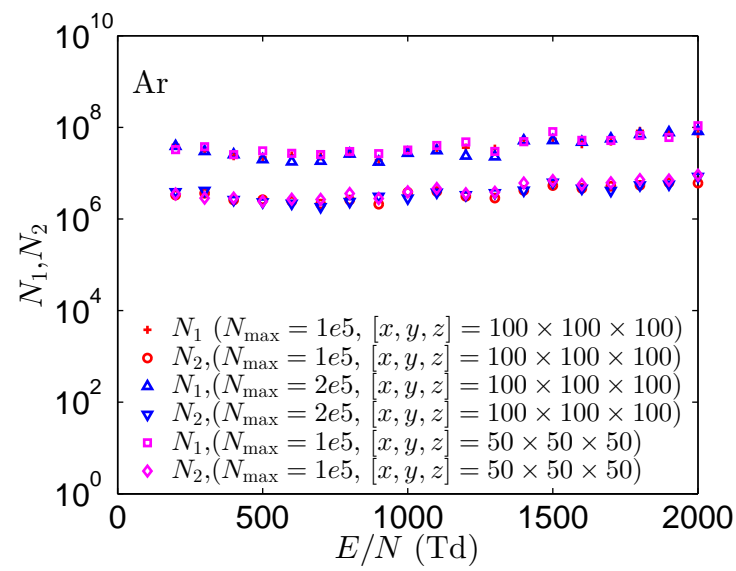

Figure 6: Electron numbers $N_{1}$ at transition 1 and $N_{2}$ at transition 2 vs $E / N$ in argon at $p=100 \mathrm{kPa}$ and room temperature for different numerical settings.

\section{References}

[1] Fridman A Chirokov A and Gutsol A 2004 Non-thermal atmospheric pressure discharge J. Phys. D: Apply. Phys. 38 R1-R24

[2] Rakov V A and Uman M A 2003 Lightning: Physics and effects Cambridge University Press. ISBN 0521583276 
[3] Raether H 1964 Electron avalanches and breakdwon in gases Butterworths London

[4] Kuffel E Zaengl W S and Kuffel J 2000 High Voltage Engineering Fundamentals (Second Edition) Elsevier Ltd. ISBN 978-0-7506-3634-6

[5] Sauli F 2014 Gaseous Radiation Detectors: Fundamentals and Applications Cambridge University Press ISBN 978-0-7506-3634-6

[6] Brook M and Kitagawa N 1960 Some aspects of lightning activity and related meteorological conditions J. Geophys. Res. 651203

[7] Franz R C Nemzek R J and Winckler J R 1990 Television Image of a Large Upward Electrical Discharge Above a Thunderstorm System Science 24948

[8] Christophorou L G 1988 Insulating gases Nucl. Instrum. Meth. A 268424

[9] Franck C M Dahl D A Rabie M Haefliger P and Koch M 2014 An Efficient Procedure to Identify and Quantify New Molecules for Insulating Gas Mixtures Contr. Plasma Phys. 543

[10] Camarri P Cardarelli R Di Ciaccio A and Santonico R 1998 Streamer suppression with SF6 in RPCs operated in avalanche mode Nucl. Instrum. Meth. A 414317

[11] Cardarelli R Di Stante L Liberti B Paolozzi L Pastori E and Santonico R 2014 New RPC gas mixtures for large area apparatuses J. of Instrum. 9 C11003

[12] Pancheshnyi S V Starikovskaia S M and Starikovskii A Yu 2000 Role of photoionization processes in propagation of cathode-directed streamer J. Phys. D: Appl. Phys. 34105

[13] Raether H 1939 The development of electron avalanche in a spark channel (from observations in a cloud chamber) Z. Phys. 112464

[14] Meek J M 1940 A Theory of Spark Discharge Phys. Rev. 57722

[15] Pedersen A McAllister I W Crichton G C and Vibholm S 1984 Formulation of the streamer breakdown criterion and its application to strongly electronegative gaes and gas mixtures Archiv für Elektrotechnik 67395

[16] Montijn C and Ebert U 2006 Diffusion correction to the RaetherMeek criterion for the avalancheto-streamer transition J. Phys. D: Appl. Phys. 392979

[17] Li C Teunissen J Nool M Hundsdorfer W and Ebert U 2012 A comparison of 3D particle, fluid and hybrid simulations for negative streamers Plasma Sources Sci. Technol. 21055019

[18] Li C Ebert U and Hundsdorfer W 2012 Spatially hybrid computations for streamer discharges: II. Fully 3D simulations J. Comput. Phys. 2311020

[19] Kline L E and Siambis J G 1972 Computer Simulation of Electrical Breakdown in Gases; Avalanche and Streamer Formation Phys. Rev. A 5794

[20] Kunhardt E E and Tzeng Y 1986 Monte Carlo Technique for Simulating the Evolution of an Assembly of Particles Increasing in Numbers J. Comput. Phys. 67279

[21] Kunhardt E E and Tzeng Y 1988 Development of an electron avalanche and its transition into streamers Phys. Rev. A 381410

[22] Dowds B J P Barrett R K and Diver D A 2003 Streamer initiation in atmospheric pressure gas discharges by direct particle simulation Phys. Rev. E 68026412

[23] Vahedi V and Surendra M 1995 A Monte Carlo Collision Model for the Particle-In-Cell Method: Applications to Argon and Oxygen Discharges Comp. Phys. Commun. 87 179-198

[24] Moss G D Pasko V P Liu N and Veronis G 2006 Monte Carlo model for analysis of thermal runaway electrons in streamer tips in transient luminous events and streamer zones of lightning leaders J. Geophys. Res. 111 A02307

[25] Chanrion O and Neubert T 2008 A PIC-MCC code for simulation of streamer propagation in air J. Comput. Phys. A 2277222

[26] Chanrion O and Neubert T 2010 Production of runaway electrons by negative streamer discharges J. Geophys. Res. 115 A00E32

[27] Lippmann C and Riegler W 2004 Detailed RPC avalanche simulations Nucl. Instrum. and Meth. A 53311

[28] Koh W H and Park I-H 2009 Numerical simulation of point-to-plane corona discharge using a Monte Carlo method Vacuum $\mathbf{8 4} 550$ 
[29] Rose D V Welch D R Clark R E Thoma C Zimmerman W R Bruner N Rambo P K and Atherton B W 2011 Towards a fully kinetic 3D electromagnetic particle-in-cell model of streamer formation and dynamics in high-pressure electronegative gases Physics of Plasmas 18093501

[30] Pancheshnyi S Biagi S Bordage M C Hagelaar G J M Morgan W L Phelps A V and Pitchford L C 2012 Electron scattering cross sections and swarm parameters for low temperature plasma modeling Chem. Phys. 398148

[31] Rabie M and Franck C M 2015 METHES: A Monte Carlo collision code for the simulation of electron transport in low temperature plasmas submitted to Comp. Phys. Commun.

[32] Skullerud H R 1968 The stochastic computer simulation of ion motion in a gas subjected to a constant electric field J. Phys. D: Appl. Phys. 11567

[33] Biagi-v8.9 database www.lxcat.net retrieved on June 112015

[34] SIGLO database www.lxcat.net retrieved on July 52015

[35] Phelps database www.lxcat.net retrieved on June 112015

[36] McDaniel E W 1989 Atomic collisions: electron and photon projectiles John Wiley \& Sons ISBN 0-471-85307-0

[37] Dujko S White R D Petrovic Z Lj and Robson R E 2010 Benchmark calculations of nonconservative charged-particle swarms in dc electric and magnetic fields crossed at arbitrary angles Phys. Rev. E 81046403

[38] Trottenberg U Oosterlee C W and Schüller A 2001 Multigrid Elsevier Academic Press

[39] Montijn C Hundsdorfer W and Ebert U 2006 An adaptive grid refinement strategy for the simulation of negative streamers J. Comput. Phys. 219801

[40] Wilson C T R 1925 The Acceleration of $\beta$-particles in Strong Electric Fields such as those of Thunderclouds Proc. Cambridge Philos. Soc. 22534

[41] Dormand J R and Prince P J 1980 A family of embedded Runge-Kutta formulae J. Comp. Appl. Math. 619

[42] Gurevich A V and Zybin K P 2005 Runaway breakdown and the mysteries of lightning Physics Today 5837

[43] Pasko V P Inan U S Bell T F 1998 Spatial structure of sprites Geophysical Research Letters 25 2123

[44] Zaengl W S Yimvuthikul S and Friedrich G 1991 The temperature dependence of homogeneous field breakdown in synthetic air IEEE Trans. Electr. Insul. 26380

[45] Miners K B Mastroianni M J Sheldon and P N Wilson D P 1982 Dew points of $\mathrm{SF}_{6} / \mathrm{N}_{2}$ gas mixtures Gaseous Dielectrics III, Pergamon Press pp. 509-516 